\title{
Variational Iteration Method of Dropping Shock Response for the Suspension Spring Packaging System
}

\author{
Shuang Song ${ }^{1}$ and An-Jun Chen ${ }^{1,2}$ \\ ${ }^{1}$ Department of Packaging Engineering, Jiangnan University, Lihu Road, Wuxi 214122, China \\ ${ }^{2}$ China National Light Industry Package Quality Supervising \& Testing Center, Lihu Road, Wuxi 214122, China \\ Correspondence should be addressed to An-Jun Chen; caj62@126.com
}

Received 23 November 2014; Accepted 6 April 2015

Academic Editor: Katsuhiko Saito

Copyright (c) 2015 S. Song and A.-J. Chen. This is an open access article distributed under the Creative Commons Attribution License, which permits unrestricted use, distribution, and reproduction in any medium, provided the original work is properly cited.

\begin{abstract}
In accordance with dropping shock dimensionless cubic nonlinear dynamic equation of suspension spring system, by variational iteration method, a first-order approximate solution of the system was obtained. The nondimensional peak of displacement, the nondimensional peak of acceleration, and the dropping shock extended period were compared with the results of the Runge-Kutta method, at which relative errors were less than $4 \%$. The influence of suspension angle on peaks of response were discussed. It shows that the maximum response nondimensional acceleration decreases with decrease of the suspension angle under condition of the same nondimensional dropping shock velocity. Conditions for resonance were obtained by applying the variational iteration method, which should be avoided in the packaging design. The results provide reference for suspension spring system design.
\end{abstract}

\section{Introduction}

The damage evaluation concept proposed by Newton [1] is the foundation for present packaging design. In procedure of transportation and storage, dropping shock might lead to serious damage to product. The suspension spring system with eight springs as cushioning components performs geometric nonlinear; the absorber effect of suspension spring system is superior to linear system and is suitable for protecting high precision instrument with low fragility. Nonlinear suspension spring natural vibration was studied by $\mathrm{Wu}$ et al. [2] based on foundation displacement. For suspension spring system under rectangular pulse excitation, the threedimensional shock spectrum and the three-dimensional damage boundary were obtained by Wang and Chen [3-5].

For complexity of nonlinear systems, the numerical method is mainly used to analyze dropping shock characteristic [6-8]. The variational iteration method (VIM) [914] has been widely applied in solving kinds of nonlinear equations. For given initial approximate solution (may contain unknown parameters), a first-order approximate analytic solution is obtained, which is not limited by small parameter. Usually, the first-order approximate solution can meet action for engineering accuracy. A dynamic model with nonlinear cubic-quintic Duffing oscillators generated from the suspension spring packaging system was established, the VIM was applied to dynamic damage evaluation [14]. However, the dynamic model with nonlinear cubic-quintic Duffing oscillators is so complicated that the expression of the frequency parameter cannot be obtained clearly, the difficulty of calculation increases, and dynamic response factors analysis is inadequate.

In this paper, for the dynamic model with cubic oscillators of the suspension spring system, the VIM is applied to get first-order approximate solution of system. The analytical expressions of important parameters with more clear physical meaning such as the frequency parameter, the nondimensional peak of displacement response, the nondimensional peak of acceleration response, the dropping shock extended period, and conditions for resonance of the system were obtained.

\section{Modeling and Equations}

The dynamic model of the suspension spring packaging system is shown in Figure 1. A product is suspended in the 


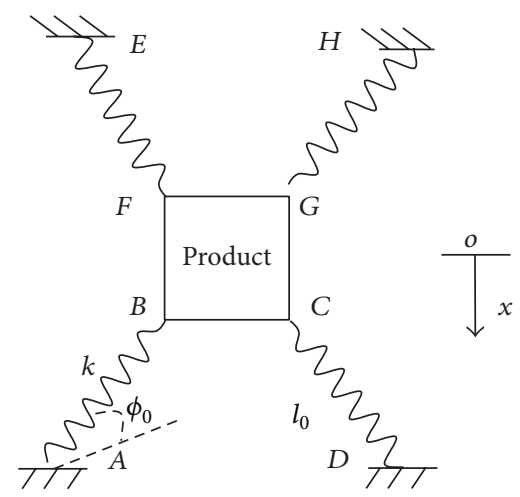

FIGURE 1: Dynamic model of the suspension spring system.

middle of the container by 8 springs (four springs are on the upside, and the other four are on the downside).

The approximate dropping shock dynamic equation [35] and dropping shock initial conditions of suspension spring system can be expressed as

$$
\begin{aligned}
m \frac{\mathrm{d}^{2} x}{\mathrm{~d} t^{2}}+8 k\left(a_{0} x+\frac{b_{0}}{l_{0}^{2}} x^{3}\right) & =0, \\
x(0) & =0, \\
\frac{\mathrm{d} x(0)}{\mathrm{d} t} & =\sqrt{2 g H},
\end{aligned}
$$

where

$$
\begin{aligned}
& a_{0}=\sin ^{2} \phi_{0}, \\
& b_{0}=\left(\frac{1}{2}\right)\left(1-6 \sin ^{2} \phi_{0}+5 \sin ^{4} \phi_{0}\right) .
\end{aligned}
$$

Here the coefficient $x$ denotes the product displacement, $k$ denotes the coupling spring stiffness coefficient, $m$ is the mass of product, $H$ is the dropping height, $\phi_{0}$ denotes the suspension angle of the system, $l_{0}$ denotes the original length of springs, and $g$ is the acceleration of gravity.

To simplify dropping shock dynamic equation, by introducing the new nondimensional parameters

$$
\begin{aligned}
& y=\frac{x}{l_{0}}, \\
& \tau=\frac{t}{T}
\end{aligned}
$$

the frequency parameter of the system is defined as

$$
\omega=\sqrt{\frac{8 k}{m}}
$$

and the period parameter of the system is defined as

$$
T=\frac{1}{\omega} .
$$

Substituting all of parameters defined above into (1), the nondimensional form of the motion equation can be written in the following form:

$$
\frac{\mathrm{d}^{2} y}{\mathrm{~d} \tau^{2}}+a_{0} y+b_{0} y^{3}=0 .
$$

Equation (10) is the nondimensional shock dynamic equation. Initial conditions can be written as

$$
\begin{aligned}
y(0) & =0, \\
\frac{\mathrm{d} y(0)}{\mathrm{d} \tau} & =V=\sqrt{\frac{2 g m H}{8 k l_{0}^{2}},}
\end{aligned}
$$

where $V$ denotes the nondimensional dropping velocity.

By (10), the nondimensional dropping velocity and the suspension angle are related to the response of the system closely, which is characterized of geometric nonlinearity.

\section{Variational Iteration Method}

VIM [9] has been widely applied in solving different kinds of nonlinear equations and is especially effective in solving nonlinear vibration problems with approximation. For the nondimensional dropping shock dynamic equation (10) and the initial conditions (11) and (12), the initial solution can be taken as below:

$$
y_{0}(\tau)=\left(\frac{V}{\alpha}\right) \sin \alpha \tau,
$$

where $\alpha$ is the frequency parameter. By using the view of VIM, we can construct the following iteration formula:

$$
\begin{gathered}
y_{n+1}(\tau)=y_{n}(\tau)+\int_{0}^{\tau} \frac{1}{\sqrt{a_{0}}} \sin \left[\sqrt{a_{0}}(s-\tau)\right] \\
\cdot\left(\frac{\mathrm{d}^{2} y_{n}(s)}{\mathrm{d} s^{2}}+a_{0} y_{n}(s)+b_{0} y_{n}^{3}(s)\right) \mathrm{d} s .
\end{gathered}
$$

The first-order iteration approximate solution is obtained as

$$
\begin{gathered}
y_{1}(\tau)=\frac{3 b_{0} V^{3}}{4 \alpha^{3}\left(\alpha^{2}-a_{0}\right)} \sin \alpha \tau-\frac{b_{0} V^{3}}{4 \alpha^{3}\left(9 \alpha^{2}-a_{0}\right)} \\
\cdot \sin 3 \alpha \tau+\left[\frac{V}{\sqrt{a_{0}}}-\frac{3 b_{0} V^{3}}{4 \sqrt{a_{0}} \alpha^{2}\left(\alpha^{2}-a_{0}\right)}\right. \\
\left.+\frac{3 b_{0} V^{3}}{4 \sqrt{a_{0}} \alpha^{2}\left(9 \alpha^{2}-a_{0}\right)}\right] \sin \sqrt{a_{0}} \tau .
\end{gathered}
$$

In order to ensure that no secular terms appear in the next iteration, let the coefficient of $\sin \sqrt{a_{0}} \tau$ be equal to zero, and the frequency parameter can be expressed as

$$
\alpha=\frac{\sqrt{5 a_{0}+\sqrt{16 a_{0}^{2}+54 b_{0} V^{2}}}}{3} .
$$


For the nondimensional shock dynamic equation (10), the first-order approximation solutions can be written as follows:

$$
\begin{aligned}
y_{1}(\tau)= & \frac{3 b_{0} V^{3}}{4 \alpha^{3}\left(\alpha^{2}-a_{0}\right)} \sin \alpha \tau \\
& -\frac{b_{0} V^{3}}{4 \alpha^{3}\left(9 \alpha^{2}-a_{0}\right)} \sin 3 \alpha \tau, \\
\frac{\mathrm{d}^{2} y_{1}(\tau)}{\mathrm{d} \tau^{2}}= & -\frac{3 b_{0} V^{3}}{4 \alpha\left(\alpha^{2}-a_{0}\right)} \sin \alpha \tau \\
& +\frac{9 b_{0} V^{3}}{4 \alpha\left(9 \alpha^{2}-a_{0}\right)} \sin 3 \alpha \tau .
\end{aligned}
$$

During product packaging design procedure, the maximum of displacement response, and the maximum of acceleration response are comparatively important for product dropping shock damage evaluation, however for general nonlinear systems getting their analytic expressions are difficult. By the first-order approximation solutions (17) and (18), when $\alpha \tau=\pi / 2$, the nondimensional peak of displacement and the nondimensional peak of acceleration can be written as

$$
\begin{aligned}
\left(y_{1}(\tau)\right)_{m} & =\left|\frac{3 b_{0} V^{3}}{4 \alpha^{3}\left(\alpha^{2}-a_{0}\right)}+\frac{b_{0} V^{3}}{4 \alpha^{3}\left(9 \alpha^{2}-a_{0}\right)}\right|, \\
\left(\frac{\mathrm{d}^{2} y_{1}(\tau)}{\mathrm{d} \tau^{2}}\right)_{m} & =\left|\frac{3 b_{0} V^{3}}{4 \alpha\left(\alpha^{2}-a_{0}\right)}+\frac{9 b_{0} V^{3}}{4 \alpha\left(9 \alpha^{2}-a_{0}\right)}\right| .
\end{aligned}
$$

The nondimensional dropping shock extended period can be obtained as

$$
\tau=\frac{\pi}{\alpha} .
$$

From (16), (19), (20) and (21), we know the nondimensional peak of displacement, the nondimensional peak of acceleration, and the nondimensional dropping shock extended period are related to $V, a_{0}$, and $b_{0} . V$ is related to $H$, and parameters $a_{0}$ and $b_{0}$ are just related to $\phi_{0}$ (the design parameter of the system). For given suspension spring system, by using (19), (20) and (21), the relations of the shock response peak and the shock extended period can be obtained for the different dropping height. We can also analyze the suspension angle influence expediently. The firstorder approximation solutions (17) and (18) have explicit physical significance, concise forms and can be analyzed easily.

\section{Numerical Results}

For the following amounts: $l_{0}=0.075 \mathrm{~m}, k=2 \times 10^{5} \mathrm{~N} / \mathrm{m}$, $H=0.5 \mathrm{~m}$ and $\phi_{0}=60^{\circ}$. As shown in Figures 2 and 3 , the nondimensional dropping shock response displacement and acceleration of the system are calculated and compared with the numerical integration solutions using the RungeKutta (ODE45) method, showing good agreement. From (19), (20) and (21), the nondimensional peak of displacement

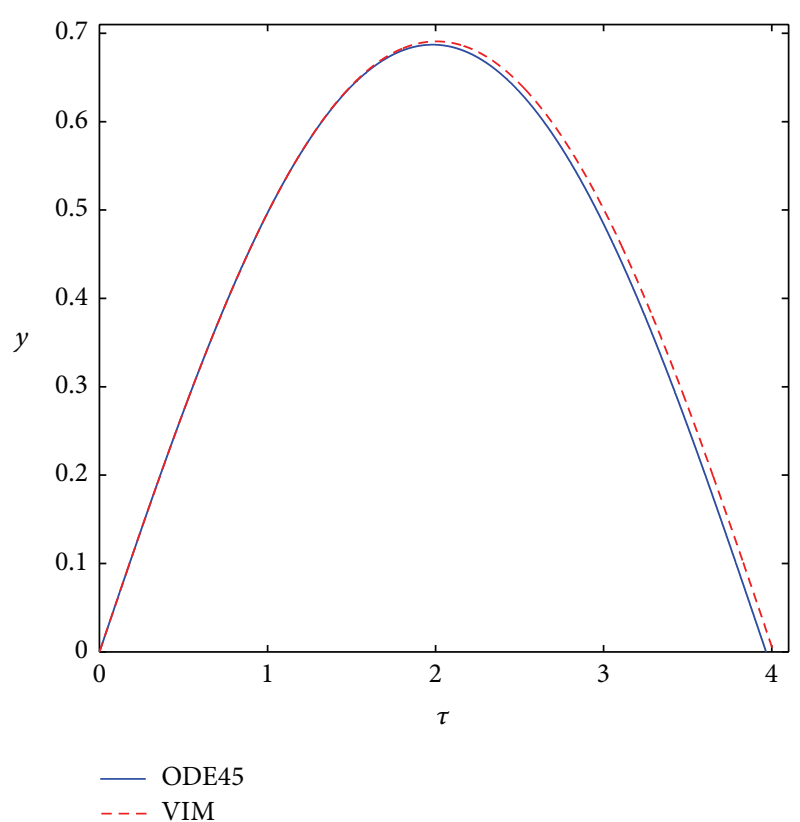

FIGURE 2: Comparison of the nondimensional displacement $y$-time $\tau$ response of the system by the VIM with the one by the Runge-Kutta (ODE45) method when the suspension angle $\phi_{0}=60^{\circ}$.

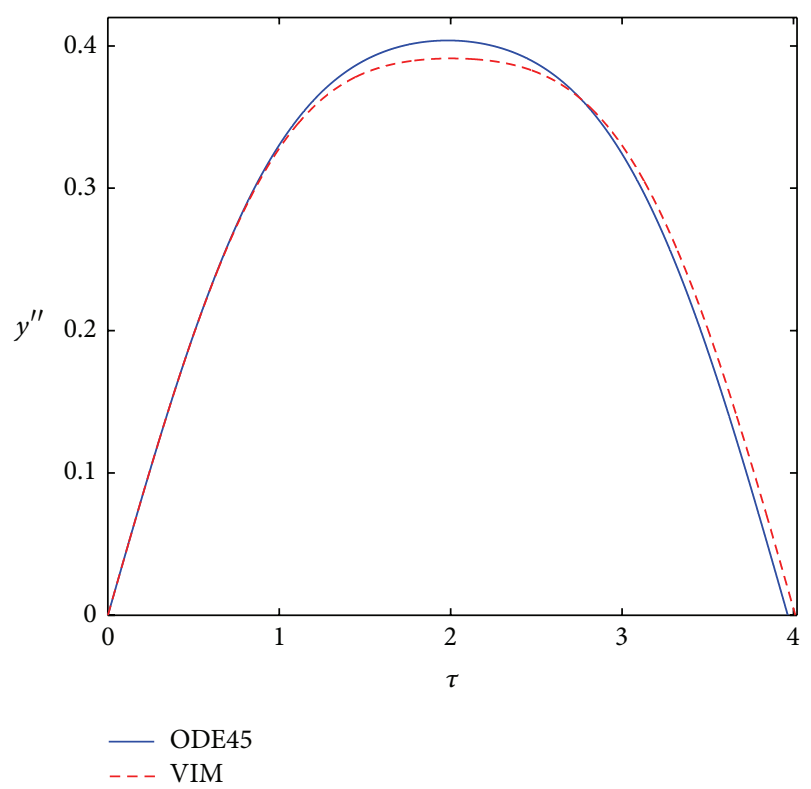

FIgURE 3: Comparison of the nondimensional acceleration $y^{\prime \prime}$-time $\tau$ response of the system by the VIM with the one by the Runge-Kutta (ODE45) method when the suspension angle $\phi_{0}=60^{\circ}$.

$y_{1 m}=0.6908$, the nondimensional peak of acceleration $\ddot{y}_{1 m}=0.3912$, and the nondimensional dropping shock extended period $\tau_{1}=4.0109$. With numerical integration solutions using ordinary differential equation-solver in MATLAB, the results are $y_{m}=0.6871, \ddot{y}_{m}=0.4038$ and $\tau=$ 3.9666. Respectively, Compared with numerical integration solutions (ODE45), the VIM results relative errors are $0.54 \%$, 


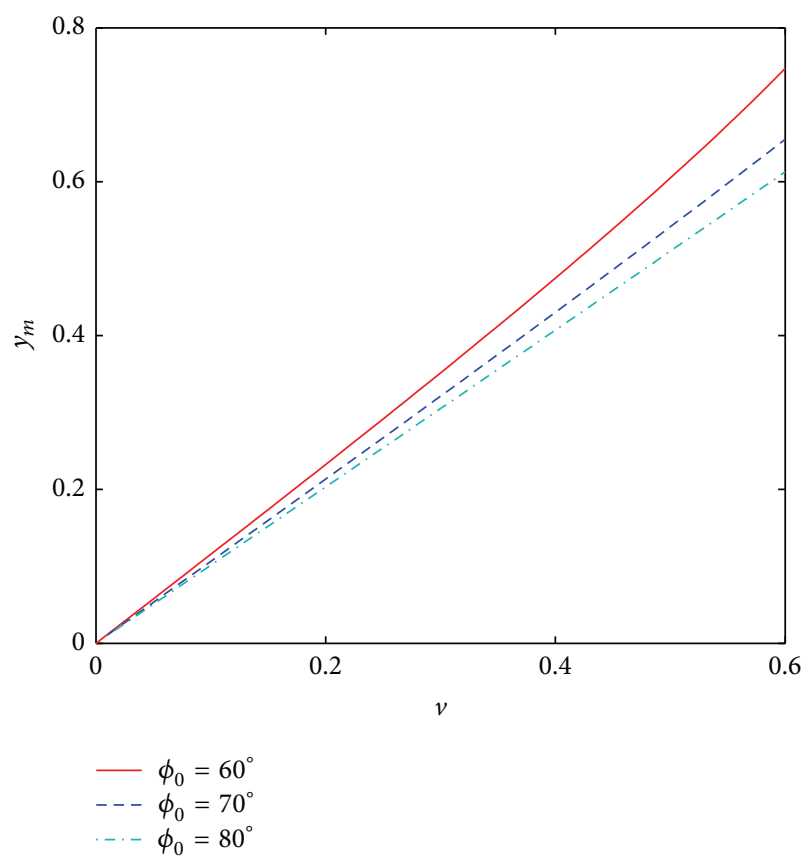

FIGURE 4: Maximum response nondimensional displacement of the system when the suspension angle $\phi_{0}=60^{\circ}, 70^{\circ}, 80^{\circ}$.

$3.12 \%$ and $1.12 \%$. The first-order approximation solutions can meet requirement of product packaging design.

In the engineering application, analyzing the main factors affecting the maximum response of the system is very significant. According to (16), (19) and (20), we can find that the nondimensional dropping shock velocity and the suspension angle are the key factors affecting the maximum response. Respectively, we choose $\phi_{0}$ equal to $60^{\circ}, 70^{\circ}$ and $80^{\circ}$. Figure 4 indicates that the maximum response nondimensional displacement of the system increases with the increase of nondimensional dropping shock velocity, and increases with the decrease of the suspension angle under condition of the same nondimensional dropping shock velocity. Figure 5 indicates that the maximum response nondimensional acceleration of the system increases with the increase of the nondimensional dropping shock velocity, and decreases with the decrease of the suspension angle under condition of the same nondimensional dropping shock velocity.

\section{Resonance}

In a cushioning packaging system, any small vibration might lead to serious damage due to inner-resonance. The innerresonance is the key problem to optimal design [12]. By (17) and formula (16), the resonance can be expected when one of the following conditions is met:

$$
\alpha=\frac{\sqrt{5 a_{0}+\sqrt{16 a_{0}^{2}+54 b_{0} V^{2}}}}{3}
$$

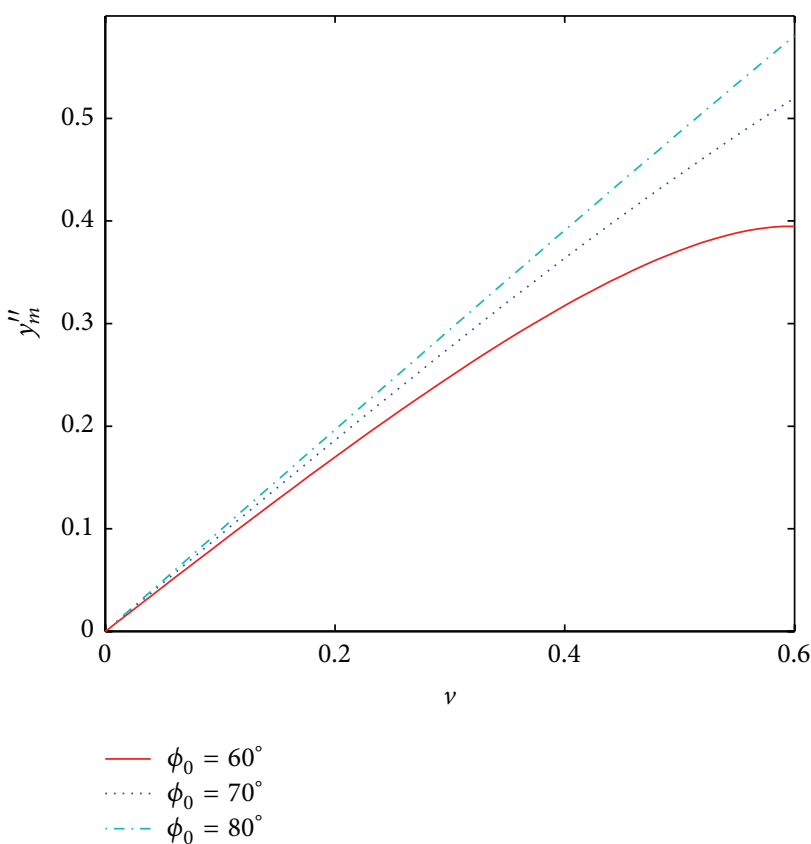

FIGURE 5: Maximum response nondimensional acceleration of the system when the suspension angle $\phi_{0}=60^{\circ}, 70^{\circ}, 80^{\circ}$.

$$
\begin{aligned}
& \alpha=\sqrt{a_{0}}, \\
& \alpha=\frac{\sqrt{a_{0}}}{3} .
\end{aligned}
$$

To verify the inner-resonance phenomenon, for the nondimensional form of the motion equation (10) and the initial conditions (11) and (12), under the condition of harmonic excitation, the dynamic equation of the system can be established as follows:

$$
\frac{\mathrm{d}^{2} y}{\mathrm{~d} \tau^{2}}+a_{0} y+b_{0} y^{3}=\beta \sin (\omega \tau)
$$

where $\beta$ is the nondimensional excitation amplitude and $\omega$ is the frequency parameter of the harmonic excitation.

We choose $\phi_{0}=65^{\circ}, \beta=0.1$, and $V=0.1$. When an external excitation frequency $\omega$ is equal to type (22), (23) and (24), the system acceleration response, respectively, as shown in Figures 6(a), 6(b), and 6(c). Figure 6 shows that the frequency of the exciting force to the system natural frequency, the system acceleration response amplitude regularly and alternately increased and reduced, known as the clapfrequency vibration phenomenon. Peak acceleration increase may cause product damage, which is dangerous for products. So inner-resonance should be avoided during cushioning packaging design procedure.

\section{Conclusion}

For the nondimensional dropping shock dynamic equation of the suspension spring system, first-order approximate analytical solutions of the nondimensional displacement and acceleration are obtained by He's variational iteration 


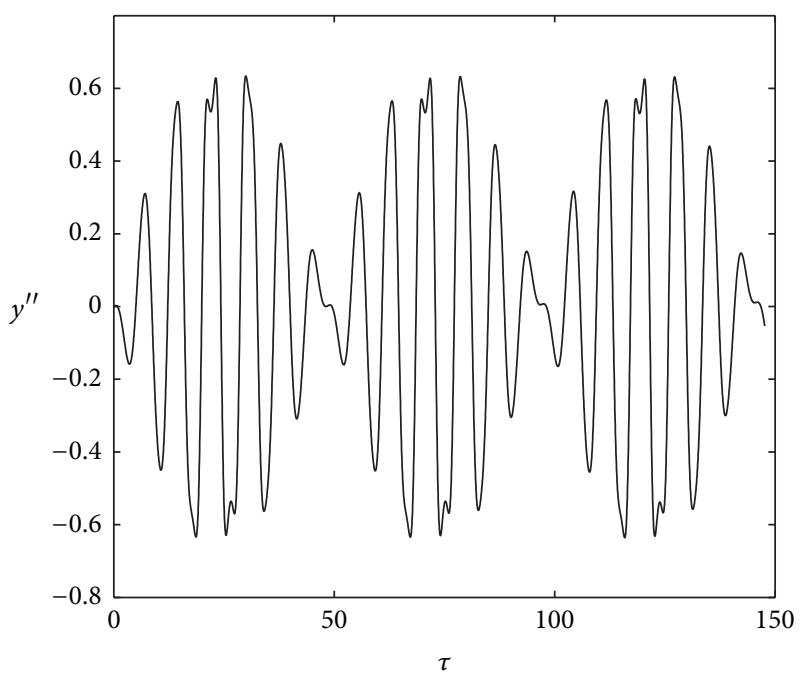

(a) $\omega=\sqrt{5 a_{0}+\sqrt{16 a_{0}^{2}+54 b_{0} V^{2}} / 3}$

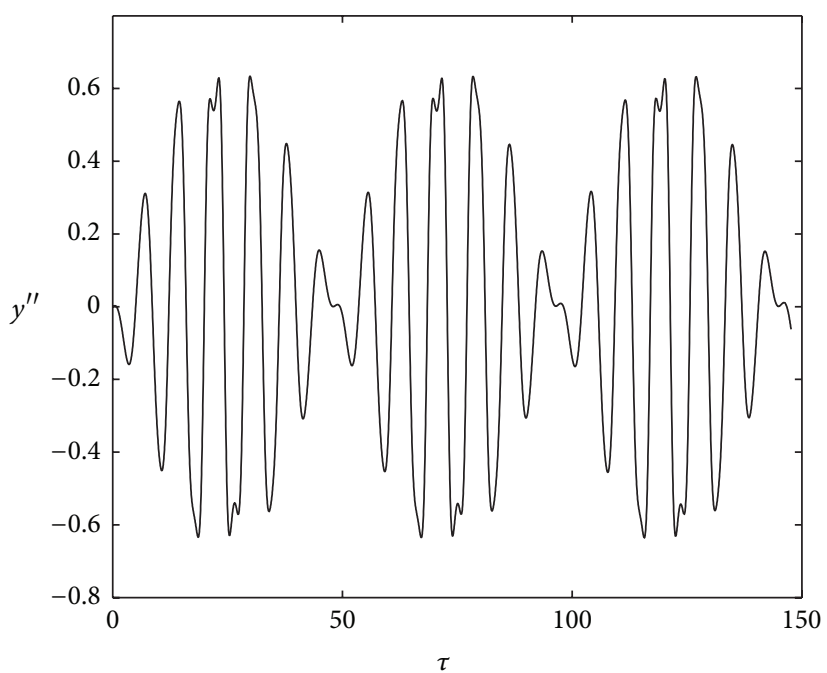

(b) $\omega=\sqrt{a_{0}}$

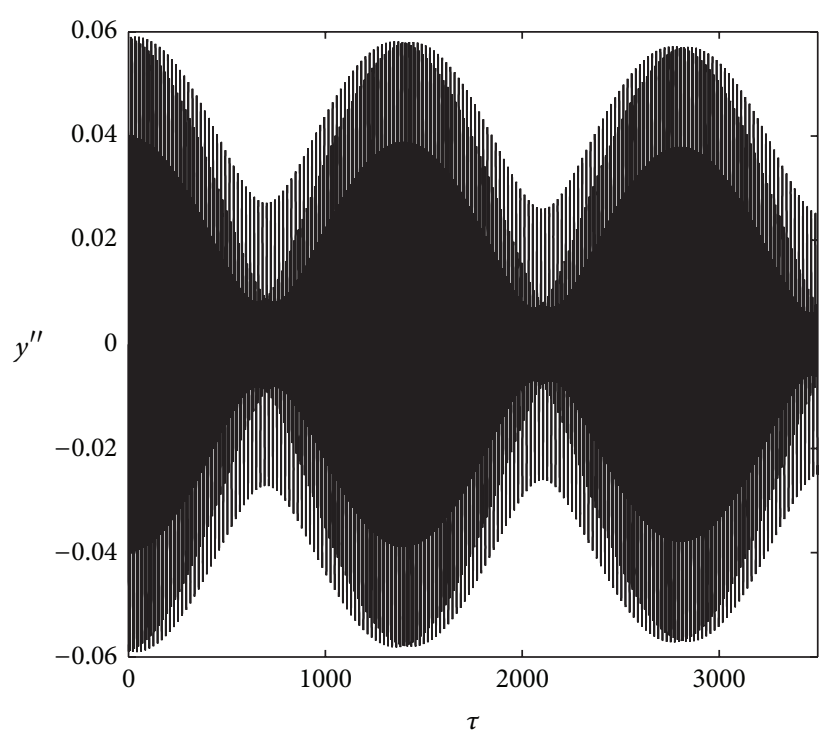

(c) $\omega=\sqrt{a_{0}} / 3$

FIGURE 6: The nondimensional acceleration response of the system under conditions for resonance.

method. Expressions of the nondimensional peak of displacement, the nondimensional peak of acceleration, and the nondimensional dropping shock extended period are obtained. Compared with the results of the Runge-Kutta method, relative errors are less than $4 \%$. The influence of dropping height and suspension angle on the nondimensional displacement and the acceleration peak values was discussed. It shows that decreasing suspension angle appropriately the nondimensional acceleration peak value would decrease, while the nondimensional displacement peak value would increase. Conditions for resonance, which should be avoided in the product packaging design procedure, can be obtained. The results provide reference for suspension spring system design.

\section{Conflict of Interests}

The authors declare that there is no conflict of interests regarding the publication of this paper.

\section{References}

[1] R. E. Newton, Fragility Assessment Theory and Practice, Monterey Research Laboratory, Monterey, Calif, USA, 1968.

[2] X. Wu, Y. X. Luo, L. J. Yang et al., "Nonlinear suspension spring natural vibration based on foundation displacement," Journal of University of Science and Technology Beijing, vol. 29, no. 12, pp. 1041-1043, 2009.

[3] L. Wang and A.-J. Chen, "Shock characteristics of a suspension spring system under action of a rectangular pulse," Journal of Vibration and Shock, vol. 31, no. 11, pp. 142-144, 2012. 
[4] L. Wang and A. J. Chen, "The damage boundary curve of the suspension packaging system under rectangular pulse," Applied Mechanics and Materials, vol. 105-107, pp. 70-73, 2012.

[5] L. Wang and A. J. Chen, "The shock response spectra of the suspension packaging system under rectangular pulse," Journal of Applied Packaging Research, vol. 5, no. 4, pp. 237-246, 2011.

[6] Y. Q. Wang and K. H. Low, "Damped response analysis of nonlinear cushion systems by a linearization method," Computers and Structures, vol. 83, no. 19-20, pp. 1584-1594, 2005.

[7] J. Wang, J.-H. Jiang, L.-X. Lu, and Z.-W. Wang, "Dropping damage evaluation for a tangent nonlinear system with a critical component," Computers and Mathematics with Applications, vol. 61, no. 8, pp. 1979-1982, 2011.

[8] Z.-W. Wang and J.-H. Jiang, "Evaluation of product dropping damage based on key component," Packaging Technology and Science, vol. 23, no. 4, pp. 227-238, 2010.

[9] J. H. He and X. H. Wu, "Variational iteration method: new development and application," Computer and Mathematics with Applications, vol. 54, no. 7-8, pp. 881-894, 2007.

[10] M. Dehghan and F. Shakeri, "Application of He's variational iteration method for solving the Cauchy reaction-diffusion problem," Journal of Computational and Applied Mathematics, vol. 214, no. 2, pp. 435-446, 2008.

[11] Y. Khan, M. Madani, A. Yildirim, M. A. Abdou, and N. Faraz, "A new approach to Van der Pol's oscillator problem," Zeitschrift für Naturforschung A, vol. 66, no. 10-11, pp. 620-624, 2011.

[12] J. Wang, Y. Khan, R.-H. Yang, L.-X. Lu, Z.-W. Wang, and N. Faraz, "A mathematical modelling of inner-resonance of tangent nonlinear cushioning packaging system with critical components," Mathematical and Computer Modelling, vol. 54, no. 11-12, pp. 2573-2576, 2011.

[13] J. Wang, Z. W. Wang, F. Duan, L. X. Lu, D. Gao, and A. J. Chen, "Dropping shock response of corrugated paperboard cushioning packaging system," Journal of Vibration and Control, vol. 19, no. 3, pp. 336-340, 2013.

[14] S. Song, N.-N. Duan, and A.-J. Chen, "Application of variational iteration method for dropping damage evaluation of the suspension spring packaging system," Abstract and Applied Analysis, vol. 2014, Article ID 385404, 7 pages, 2014. 

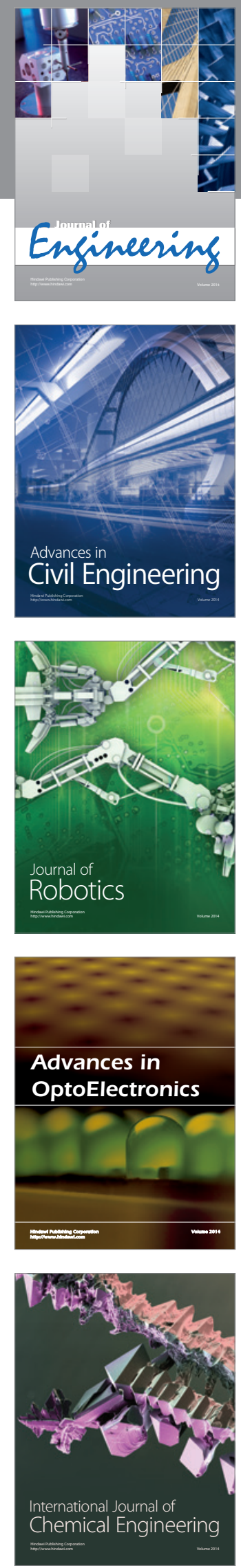

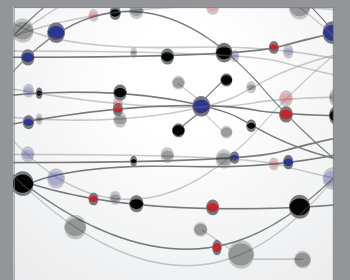

The Scientific World Journal
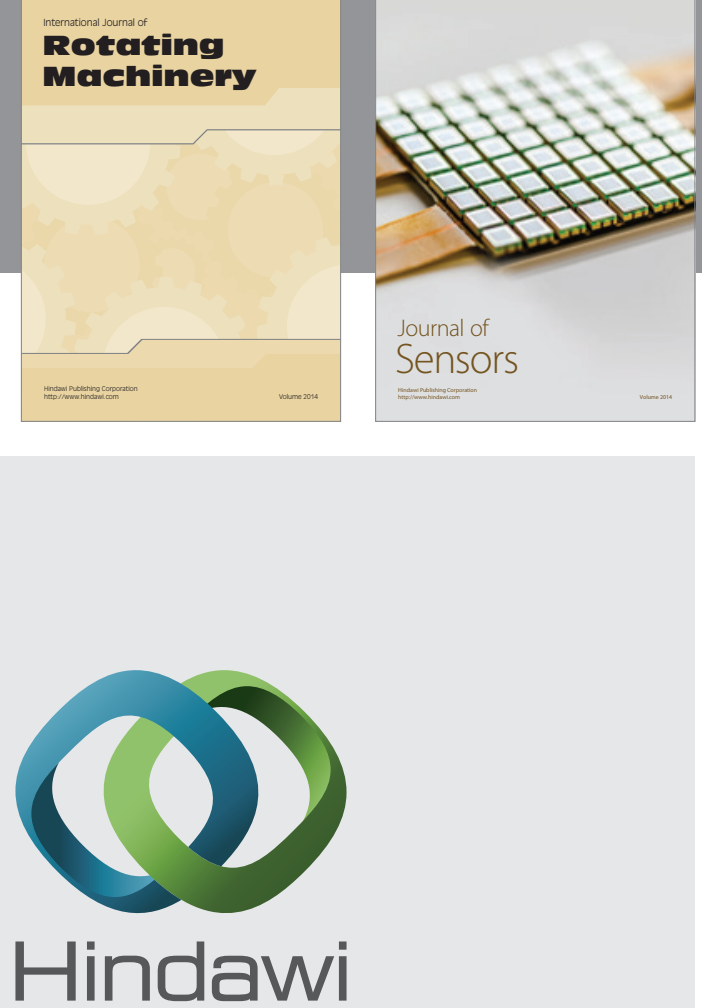

Submit your manuscripts at http://www.hindawi.com
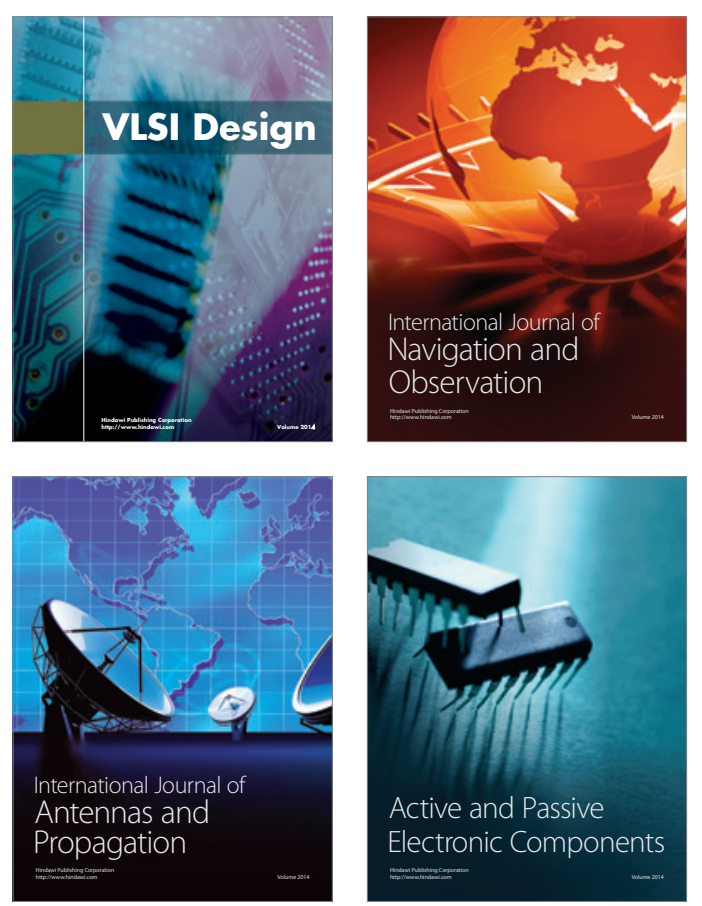
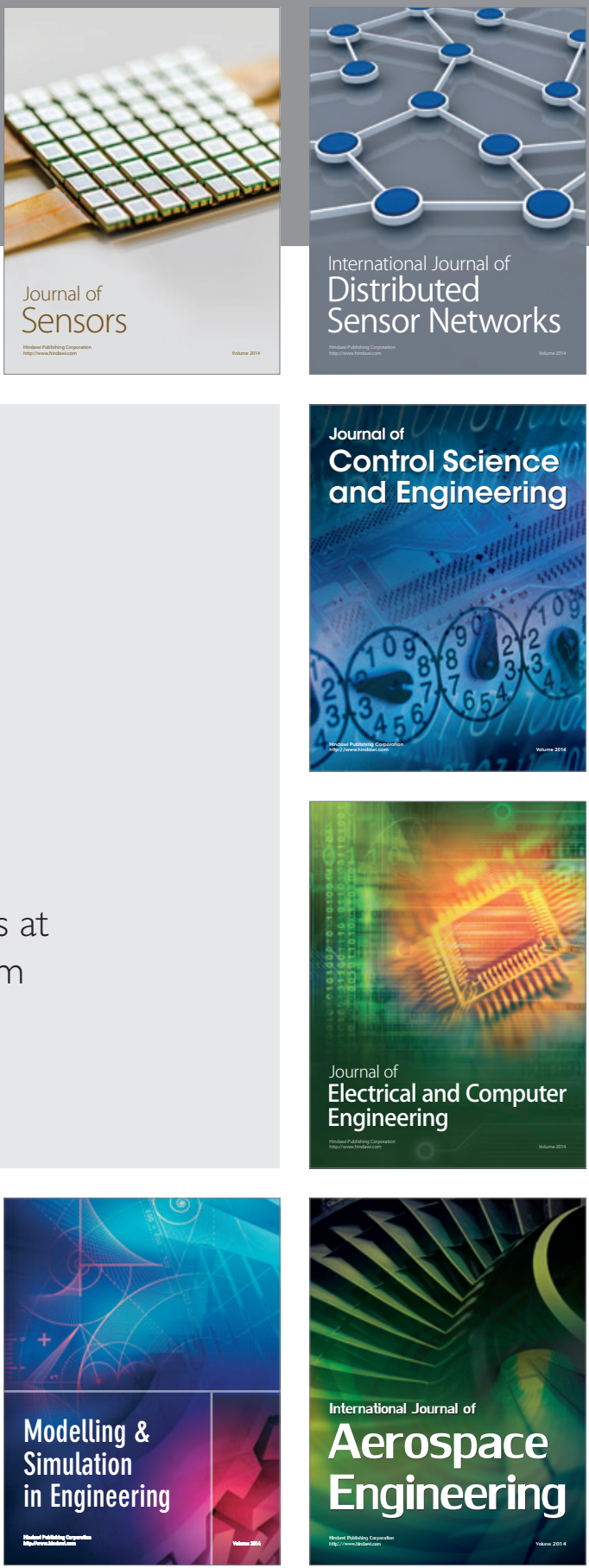

Journal of

Control Science

and Engineering
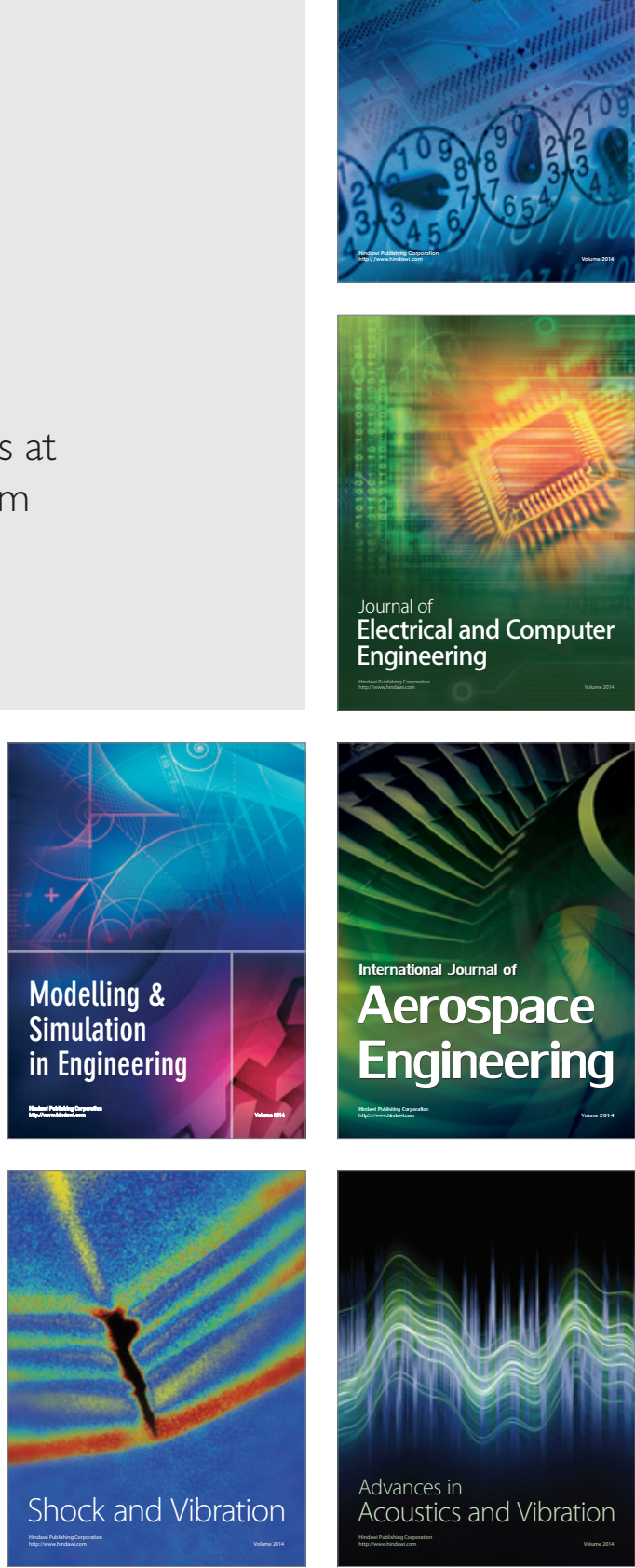